Case Report

\title{
Sorafenib-Associated Heart Failure Complicated by Cardiogenic Shock after Treatment of Advanced Stage Hepatocellular Carcinoma: A Clinical Case Discussion
}

\author{
Candace $\mathrm{Wu}^{1}$ and Kamal Shemisa ${ }^{2}$ \\ ${ }^{1}$ University of Texas Southwestern Medical Center, Dallas, TX, USA \\ ${ }^{2}$ Division of Cardiology, Department of Internal Medicine, University of Texas Southwestern Medical Center, Dallas, TX, USA
}

Correspondence should be addressed to Candace Wu; candace.wu@utsouthwestern.edu

Received 1 November 2016; Accepted 13 April 2017; Published 27 April 2017

Academic Editor: Kuan-Rau Chiou

Copyright (c) 2017 Candace Wu and Kamal Shemisa. This is an open access article distributed under the Creative Commons Attribution License, which permits unrestricted use, distribution, and reproduction in any medium, provided the original work is properly cited.

\begin{abstract}
Background. Sorafenib, an oral tyrosine kinase inhibitor (TKI), targets multiple tyrosine kinase receptors (TKRs) involved in angiogenesis and tumor growth. Studies suggest that inhibition of TKR impacts cardiomyocyte survival. Inhibition of VEGF signaling interrupts angiogenesis and is associated with the development of hypertension and compensatory hypertrophy. Compensated hypertrophy ultimately leads to heart failure. Case Description. A 76-year-old man with a past medical history of systolic heart failure due to ischemic cardiomyopathy and stage IIIC hepatocellular carcinoma (HCC) presented with symptoms of decompensated heart failure. Four months prior to admission, he was started on sorafenib. Results. Our patient was treated with intravenous furosemide and guideline directed therapy. Clinical status was complicated by the development of low cardiac output and shock requiring inotropic support. Careful titration of heart failure medication led to hemodynamic improvement and discontinuation of dobutamine. Conclusion. Greater awareness of sorafenib cardiotoxicity is essential. As TKI usage grows for treatment of cancers, heart failure-related complications will increase. In our patient, routine heart failure management and cessation of sorafenib led to clinical improvement. Future studies on the treatment of sorafenib cardiotoxicity should be explored further in this unique patient population.
\end{abstract}

\section{Introduction}

Sorafenib is an oral multikinase inhibitor that targets pathways involved in angiogenesis and tumor growth by inhibiting tyrosine kinase receptors and is recommended as a first-line drug for the treatment of advanced hepatocellular carcinoma due to limited alternatives. The effect of sorafenib on the human body is not limited to hepatocellular cancer cells and has been linked to cardiovascular toxicity leading to the pathogenesis of clinical heart failure and resistant hypertension $[1,2]$.

This report presents the clinical case of a patient with ischemic cardiomyopathy and advanced HCC who was started on sorafenib and subsequently developed heart failure complicated by cardiogenic shock. We will review the clinical case, the biological basis for TKI cardiac toxicity, and the therapeutic challenges and considerations for the treatment of sorafenib-associated hypertension and heart failure.

\section{Clinical Case}

A 76-year-old man presented with worsening dyspnea at rest and on exertion, orthopnea, and lower extremity edema. The past medical history was significant for single-vessel coronary artery disease ( $\mathrm{s} / \mathrm{p}$ percutaneous coronary intervention), systolic heart failure with reduced ejection fraction (LVEF $37 \%$ ) due to ischemic cardiomyopathy, severe ventricular hypertrophy, chronic atrial fibrillation, hypertension, hyperlipidemia, diabetes mellitus type two, chronic obstructive pulmonary disease, and Stage IIIC hepatocellular carcinoma. Family history was positive for hypertension and diabetes mellitus type two. He was previously treated with guideline 


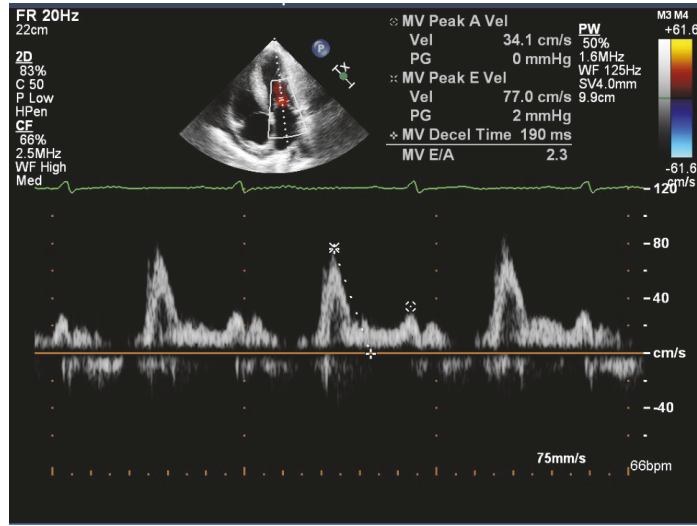

(a)

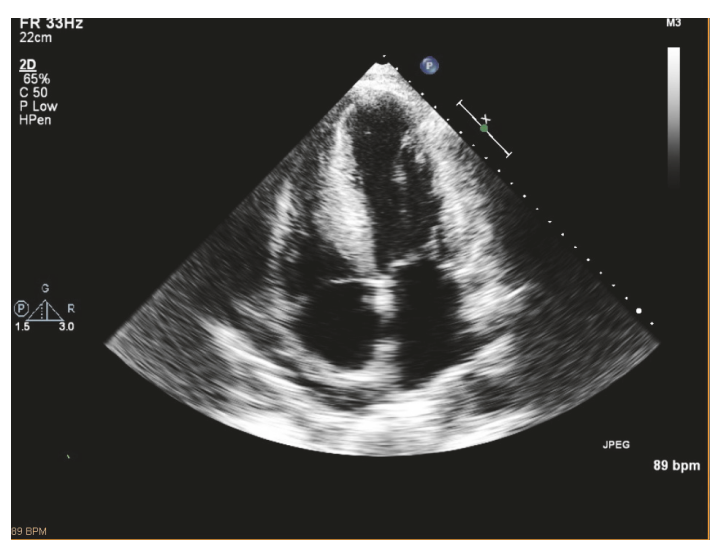

(b)

FIGURE 1: (a) At baseline, the transthoracic echocardiogram demonstrates moderately depressed LV function (LVEF-40\%) with significant concentric and septal LV hypertrophy (LV mass 367 grams, IVSd- $2.1 \mathrm{~cm}$, PWT-1.5 cm, RWT-0.67). (b) There is further evidence of elevated filling pressures. There is left atrial enlargement (LA dimension- $4.6 \mathrm{~cm}$ ), mildly elevated estimated right ventricular systolic pressure (RVSP $36 \mathrm{mmHg}$ ), and restrictive filling on mitral inflow Doppler (E/A-2.3, deceleration time 190 milliseconds).

TABLE 1: The pertinent medication list and dose.

\begin{tabular}{lc}
\hline Medication & Dose \\
\hline Sorafenib & $200 \mathrm{mg}$ by mouth twice daily \\
Carvedilol & $3.125 \mathrm{mg}$ by mouth twice daily \\
Furosemide & $80 \mathrm{mg}$ by mouth twice daily \\
Lisinopril & $10 \mathrm{mg}$ by mouth daily \\
Aspirin & $81 \mathrm{mg}$ by mouth daily \\
Warfarin & $2.5 \mathrm{mg}$ by mouth daily \\
Nitroglycerin & $0.4 \mathrm{mg}$ sublingual as needed \\
\hline
\end{tabular}

directed therapy for systolic heart failure (Table 1). The transthoracic echocardiogram was significant for moderately depressed left ventricular (LV) function, left atrial dilation, normal ventricular chamber dimensions, increased LV mass, and global hypokinesis (Figure 1).

Eight months prior to admission, a cardiac MRI demonstrated findings suggestive of mild inferior wall myocardial ischemia on gadolinium imaging and moderately depressed systolic dysfunction. He was treated medically for ischemic heart failure without further risk stratification. Four months prior to admission, the patient was incidentally found to have a palpable right upper quadrant mass, which, by ultrasound, revealed a large heterogeneous echogenic density (measuring $13 \mathrm{~cm}$ ). He underwent transhepatic biopsy and pathologic results were significant for poorly differentiated pleomorphic hepatocellular carcinoma. CT of the abdomen and pelvis was significant for tumor extension into the inferior vena cava and subdiaphragmatic space. A markedly elevated alphafetoprotein (AFP) level (>10,000 ng/mL) was measured, suggestive of a high tumor burden.

His WHO cancer staging was Stage IIIC based on clinical as well as imaging findings. The 1-year survival of Stage IIIC HCC is 6-8 months for patients and the recommended treatment is a tyrosine kinase inhibitor (TKI) called sorafenib, which extends median survival length to 10.7 months [3].
The patient presented to the emergency department hypertensive $(169 / 79 \mathrm{mmHg})$ and tachycardic with a ventricular rate of 127 beats per minute (Figure 2). The physical exam was consistent with volume overload, and admission labs were notable for elevated BNP, abnormal BUN/creatinine, and elevated liver function tests (Table 2). Chest X-ray demonstrated cardiomegaly with pulmonary venous congestion and a moderate-sized right-sided pleural effusion.

The clinical exam was consistent with decompensated heart failure. The patient was treated with intravenous furosemide, carvedilol, and lisinopril. After sufficient diuresis (10 liters of fluid), clinical symptoms improved minimally (Table 3). However, within the subsequent 18 hours, the patient developed persistent hypotension (MAP $60 \mathrm{mmHg}$ ), worsening renal function, and cool extremities indicative of impending cardiogenic shock. He continued to have elevation of JVP on physical exam, which suggested restrictive filling of the right ventricle (RV) as well as RV failure. A pulse wave Doppler across the LVOT suggested diminished stroke volume after calculation of the LV velocity time integral (Figure 3). A right heart catheterization was subsequently performed which showed mildly elevated right- and leftsided ventricular filling pressure, pulmonary hypertension, and low cardiac output (Table 3 ). He was then treated with continuous infusion of dobutamine and furosemide. An EKG at the time demonstrated improvement of the ventricular rate without evidence of ST-T wave changes (Figure 4).

Based on the antecedent history of recent initiation of sorafenib followed by deterioration in cardiac function, sorafenib-induced decompensated heart failure was diagnosed. This case report serves as an example of the therapeutic challenges of heart failure management in patients treated for hepatocellular carcinoma with sorafenib. The cardiac toxicity of sorafenib is known and while many patients who have preexisting heart failure are not initiated on tyrosine kinase inhibitors for this reason, limited therapies for cancers such as HCC may compel care providers to do 
TABLE 2: Admission physical exam and notable labs.

\begin{tabular}{|c|c|c|c|}
\hline \multirow{4}{*}{ Physical exam } & Vital signs & \multicolumn{2}{|c|}{ BP 169/79 mmHg, HR 129 bpm } \\
\hline & Cardiovascular & \multicolumn{2}{|c|}{$\begin{array}{l}\text { Irregularly irregular rhythm, tachycardic, JVP } 15 \mathrm{~cm} \mathrm{H}_{2} \mathrm{O} \text { with head of bed at } \\
\qquad 30 \text { degrees }\end{array}$} \\
\hline & Lungs & \multicolumn{2}{|c|}{ Symmetric chest rise with bibasilar rales } \\
\hline & Extremities & \multicolumn{2}{|c|}{$3+$ lower extremity pitting edema to the mid-shins bilaterally } \\
\hline & & Patient result & Normal \\
\hline \multirow{12}{*}{ Laboratory results } & Sodium & 139 & $135-145 \mathrm{mEq} / \mathrm{L}$ \\
\hline & Potassium & 4 & $3.6-5.0 \mathrm{mEq} / \mathrm{L}$ \\
\hline & BUN & 21 & $7-21 \mathrm{mg} / \mathrm{dL}$ \\
\hline & Creatinine & 1.48 & $<1 \mathrm{mg} / \mathrm{dL}$ \\
\hline & Magnesium & 1.6 & $1.4-1.8 \mathrm{mEq} / \mathrm{L}$ \\
\hline & $\mathrm{BNP}$ & 3298 & $<100 \mathrm{pg} / \mathrm{mL}$ \\
\hline & Troponin I & 0.05 & $<0.03$ \\
\hline & AST & 95 & $5-35 \mathrm{U} / \mathrm{L}$ \\
\hline & ALT & 60 & $7-56 \mathrm{U} / \mathrm{L}$ \\
\hline & Alkaline phosphatase & 234 & $38-126 \mathrm{U} / \mathrm{L}$ \\
\hline & Total bilirubin & 4.2 & $0.2-1.3 \mathrm{mg} / \mathrm{dL}$ \\
\hline & Procalcitonin & + & Undetectable \\
\hline
\end{tabular}

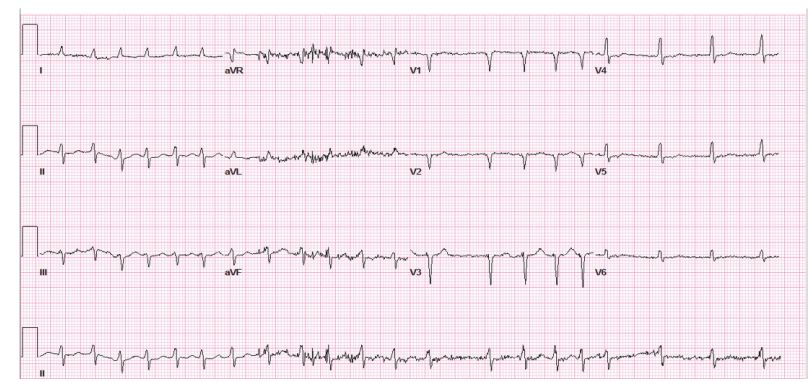

FIGURE 2: Atrial fibrillation with rapid ventricular response (ventricular rate $127 \mathrm{bpm}$ ) with evidence of prior septal infarct and nonspecific ST-T wave abnormalities in the precordium. There are low QRS voltages noted across leads likely due to chronic obstructive pulmonary disease.

so. Cautious clinical decision-making, heart failure followup, and recognition of higher risk groups in this unique subset of patients are needed. It is our goal to discuss the mechanism of sorafenib-associated cardiac toxicity and other tyrosine kinase inhibitors. We also review clinical strategies for treatment of heart failure complications.

\section{Discussion}

Sorafenib is an oral tyrosine kinase inhibitor that targets multiple tyrosine kinase receptors involved in angiogenesis and tumor growth and is recommended as a first-line drug for the treatment of advanced HCC [1]. In cancer cells, tyrosine kinase activity is increased due to mutations in the genes associated with the fundamental regulatory components of the enzyme. Mutations in cancer cells can also lead to overexpression of the tyrosine kinase genes. Tyrosine kinase inhibition is mediated through binding of a ligand or antibody (inhibitor), thus preventing the receptor from achieving its activated form. Autophosphorylation of the tyrosine kinase subsequently activates a series of protein kinase cascades in one of two dominant pathways involved in cell proliferation, hypertrophy, function, and survival. These are the phosphatidylinositol 3-kinase (PI3K) pathway via $\mathrm{AKT}$ or the mitogen-activated protein kinase (MAPK) cascade via RAS and ERK (extracellular signal regulated kinase) $[4,5]$.

The Signaling Pathway of Tyrosine Kinases, Sites of Inhibition, and Cardiotoxicity Secondary to Trastuzumab following Treatment of HER2+ Breast Cancer. To further understand the reasons for sorafenib-associated cardiotoxicity, it is relevant to discuss the lessons learned from earlier generation TKIs, the exact cellular pathways affected, and the mechanisms behind the pathogenesis of clinical heart failure. Patients with ERBB2 receptor, also known as HER2 (human epidermal growth factor receptor), breast cancer that were receiving anthracycline-based chemotherapy were 3.5 times more likely ( $8 \%$ versus $27 \%, p<0.001$ ) to develop asymptomatic cardiac dysfunction on echocardiography or clinical heart failure [6]. Clinically significant predictors for cardiac toxicity among several cohorts receiving trastuzumab were history 
TABLE 3: (a) The clinical exam and hemodynamic profile consistent with low output heart failure prior to treatment with dobutamine infusion. (b) The clinical exam and hemodynamic profile after treatment with dobutamine infusion.

(a)

\begin{tabular}{|c|c|c|c|}
\hline Physical exam & $\begin{array}{c}\text { Vital signs } \\
\text { MAP 59-62 mmHg, HR } 92 \text { bpm, } \\
\text { RR 20/min }\end{array}$ & $\begin{array}{l}\text { Right heart } \\
\text { catheterization }\end{array}$ & Measurements \\
\hline General & $\begin{array}{l}\text { Wrapped in blankets including } \\
\text { head. Appears fatigued. }\end{array}$ & RA mean pressure & $8 \mathrm{mmHg}$ \\
\hline Head, neck, throat & Dry mucous membranes. & RV pressure & $33 / 9 \mathrm{mmHg}$ \\
\hline Cardiovascular & $\begin{array}{l}\text { Irregularly irregular. Parasternal } \\
\text { holosystolic murmur. +RV gallop. } \\
\text { JVP } 20 \mathrm{~cm} \mathrm{H}_{2} \mathrm{O} \text { at } 30 \text { degrees. }\end{array}$ & PA pressure & $\begin{array}{l}32 / 23 \mathrm{mmHg} \text {, mean } \\
27 \mathrm{mmHg}\end{array}$ \\
\hline Lungs & $\begin{array}{l}\text { Coarse breath sounds. No wheezes. } \\
\text { Normal work of breathing. }\end{array}$ & PCWP & $18 \mathrm{mmHg}$ \\
\hline Abdomen & $\begin{array}{l}\text { Soft, normoactive bowel sounds. } \\
\text { Moderate to severe hepatomegaly } \\
\text { with hepatojugular reflux. }\end{array}$ & $\begin{array}{l}\text { Pulmonary vascular } \\
\text { resistance }\end{array}$ & $3.8 \mathrm{WU}$ \\
\hline Extremities & $\begin{array}{l}2+\text { pitting edema to knees. No } \\
\text { cyanosis or clubbing. }\end{array}$ & $\begin{array}{l}\text { Cardiac output }(\mathrm{L} / \mathrm{min}) \\
\quad \text { (thermal dilution) }\end{array}$ & $\begin{array}{c}3.91(\mathrm{~L} / \mathrm{min}), \mathrm{Sv} 02-53 \% \text {, } \\
\text { Hgb } 15.2\end{array}$ \\
\hline Skin & Slightly cool extremities. & Cardiac index & $1.70 \mathrm{~L} / \mathrm{min} / \mathrm{m}^{2}$ \\
\hline
\end{tabular}

(b)

\begin{tabular}{|c|c|c|c|}
\hline Physical exam & $\begin{array}{c}\text { Vital signs } \\
\text { MAP } 72 \mathrm{mmHg} \text {, HR } 85 \mathrm{bpm}, \mathrm{RR} \\
\text { 14/min }\end{array}$ & $\begin{array}{l}\text { Right heart } \\
\text { catheterization }\end{array}$ & Measurements \\
\hline General & Brighter affect. & RA mean pressure & $5 \mathrm{mmHg}$ \\
\hline Head, neck, throat & Moist mucous membranes. & $\mathrm{RV}$ pressure & $26 / 5 \mathrm{mmHg}$ \\
\hline Cardiovascular & $\begin{array}{l}\text { Irregularly irregular. Parasternal } \\
\text { soft holosystolic murmur. JVP } 8 \mathrm{~cm} \\
\mathrm{H}_{2} \mathrm{O} \text { at } 30 \text { degrees. }\end{array}$ & PA pressure & $\begin{array}{l}26 / 15 \mathrm{mmHg} \text {, } \\
\text { mean } 19 \mathrm{mmHg}\end{array}$ \\
\hline Lungs & $\begin{array}{l}\text { Clear breath sounds. No wheezes. } \\
\text { Normal work of breathing. }\end{array}$ & PCWP & $12 \mathrm{mmHg}$ \\
\hline Abdomen & $\begin{array}{l}\text { Soft, normoactive bowel sounds. } \\
\text { Moderate hepatomegaly with firm } \\
\text { liver edges, absent hepatojugular } \\
\text { reflux. }\end{array}$ & $\begin{array}{l}\text { Pulmonary vascular } \\
\text { resistance }\end{array}$ & $1.4 \mathrm{WU}$ \\
\hline Extremities & No edema, cyanosis, or clubbing. & $\begin{array}{l}\text { Cardiac output }(\mathrm{L} / \mathrm{min}) \\
\quad(\text { thermal dilution })\end{array}$ & $\begin{array}{c}5.3(\mathrm{~L} / \mathrm{min}), \mathrm{Sv} 02-75 \% \\
\mathrm{Hgb} 14.2\end{array}$ \\
\hline Skin & $\begin{array}{c}\text { Pink fingertips and warm } \\
\text { extremities. }\end{array}$ & Cardiac index & $2.4 \mathrm{~L} / \mathrm{min} / \mathrm{m}^{2}$ \\
\hline
\end{tabular}

of cardiac disease at baseline, treatment with anthracyclines, and age $>70$ years $[7,8]$.

The true incidence of heart failure secondary to trastuzumab alone was likely unknown due to treatment confounding with anthracyclines and taxanes. Assessing the true clinical risk of cardiomyopathy among patients receiving trastuzumab has therefore led to clinical trials with prespecified cardiac end points which estimate the incidence to be less frequent when administered alone (4-7\%) [9, 10]. Clinical follow-up data from these trials further demonstrated that trastuzumab cardiac toxicity is reversible shortly after discontinuation and may be responsive to medical therapy [11]. This would suggest that the severity of cardiac toxicity secondary to TKIs is most pronounced when the cardiac myocyte is simultaneously weakened by another process that creates an energy deficit, and, subsequently, the required metabolic signaling needed to maintain survival is hindered through the disruption of these critical pathways. As will be described, the degree of cardiac toxicity caused by TKIs depends on the inhibitory effect on the dominant signaling pathway of the cardiac myocyte, the selectivity of tyrosine kinase inhibition, the concomitant treatment with older cardiotoxic chemotherapeutic agents, anthracyclines or taxanes, and the duration of treatment $[10,12]$.

Tyrosine Kinase Inhibition Promotes an Energy Deficit and Interferes with Cardiac Myocyte Survival Pathways. TKIs are considered very effective in the treatment of the respective cancers but, as was described, can have deleterious effects on the cardiovascular system. Both clinical experiences followed 


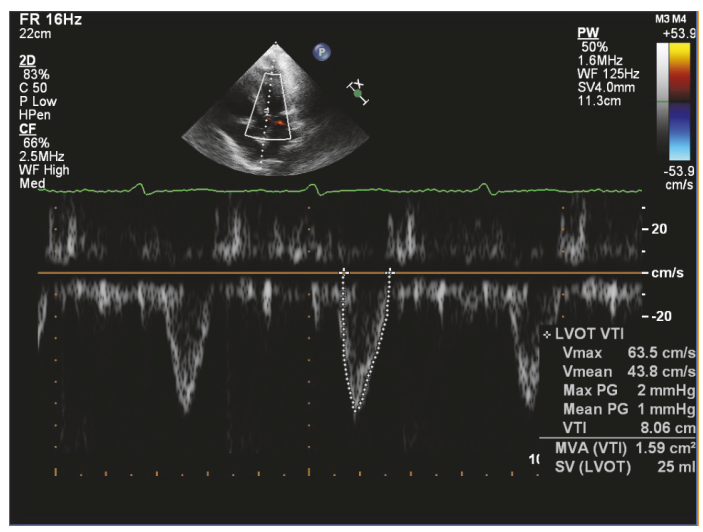

FIGURE 3: At the time of clinical deterioration, pulse Doppler of the LVOT and continuity equation calculated SV/CO (LVOT dimension$2.0 \mathrm{~cm}$, VTI- $8.1 \mathrm{~cm})$ suggested low stroke volume $(25 \mathrm{ml})$ and cardiac output $(2.22 \mathrm{~L} / \mathrm{min})$.

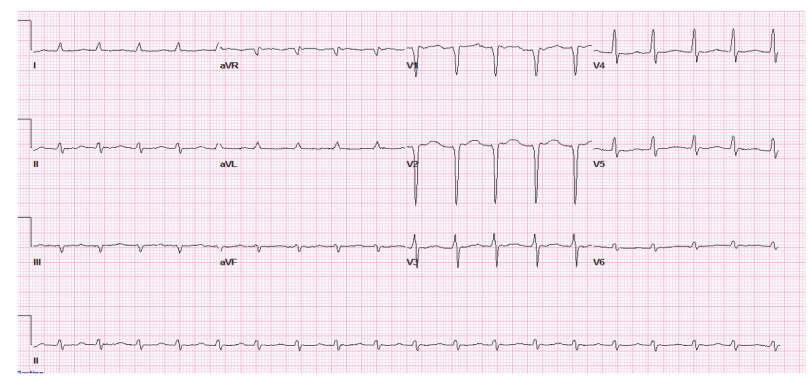

FIGURE 4: Atrial fibrillation with fairly regular R-R intervals (ventricular rate $113 \mathrm{bpm}$ ) and slower rate while on beta-blocker. The QRS morphology and voltages remain unchanged from prior tracing.

by basic investigations regarding deficient cellular signaling due to trastuzumab and bevacizumab have furthered our understanding of the importance of tyrosine kinase signaling in the pathogenesis of heart failure and hypertension in patients receiving these treatments for cancer. As newer TKIs are being developed, there is greater scrutiny for reporting adverse cardiovascular events during clinical trials. In the search for better and more effective anticancer drugs, multikinase inhibitors were developed to achieve greater inhibition of TKR and downstream signaling. The ultimate effect of less selectivity is the shutting down of signaling, ATP utilization, and the cell machinery for cell division. Sorafenib, a multikinase inhibitor, was designed for the inhibition of cell proliferation.

Studies that have elucidated the cellular mechanisms behind cardiotoxicity due to sorafenib suggest that inhibition of RAF-1 and B-RAF kinase impact cardiomyocyte survival (Figure 5) [12]. RAF-1 signaling phosphorylates a series of kinases in the MAPK group, MEK1 and ERK1, which potentiates cardiac myocyte hypertrophic growth and survival [13, $14]$.

The RAF-1 kinase also inhibits cell death pathways mediated by apoptosis signal-regulating kinase 1 (ASK1) as well as mammalian sterile 20 kinase 2 (MST2). Both ASK1 and MST2 are proapoptotic kinases and have roles in oxidant stress-induced injury. Deletions of RAF-1 in the heart result in ventricular dilation, reduced contractility, and increased cell death $[12,15]$. When mice with deleted RAF-1 are subjected to pressure overload, cardiomyocyte death was increased, indicating that RAF-1 kinase activity may be cardioprotective [16].

Nonselective TKIs such as sorafenib that target multiple kinases have great potential for intracellular toxicity. Myocardial cells require greater energy demands than other cells and therefore are particularly sensitive to deficiencies in energy production. Some case reports have indicated the potential for reversibility of sorafenib-induced cardiomyopathy after discontinuation though it is unclear to what extent or after what duration of treatment that reversibility is still feasible [17].

Mechanism of Cardiotoxicity after Treatment with Sorafenib May Be In Part due to VEGF Inhibition. TKRs were initially noted to enhance gene expression and protein synthesis involved in cardiac hypertrophy [18]. Deleterious effects on the cardiovascular system were observed with inhibition of VEGF (vascular endothelial growth factor) and PDGF (platelet derived growth factor) receptors. Inhibition of VEGF signaling with bevacizumab was shown to increase vascular tone and disrupt microvascular blood flow $[19,20]$. Clinical consequences of disrupting VEGF signaling include a higher risk for worsening hypertension and a twofold greater risk for heart failure among patients with cancers of solid tumor origin [21, 22]. Among patients undergoing 


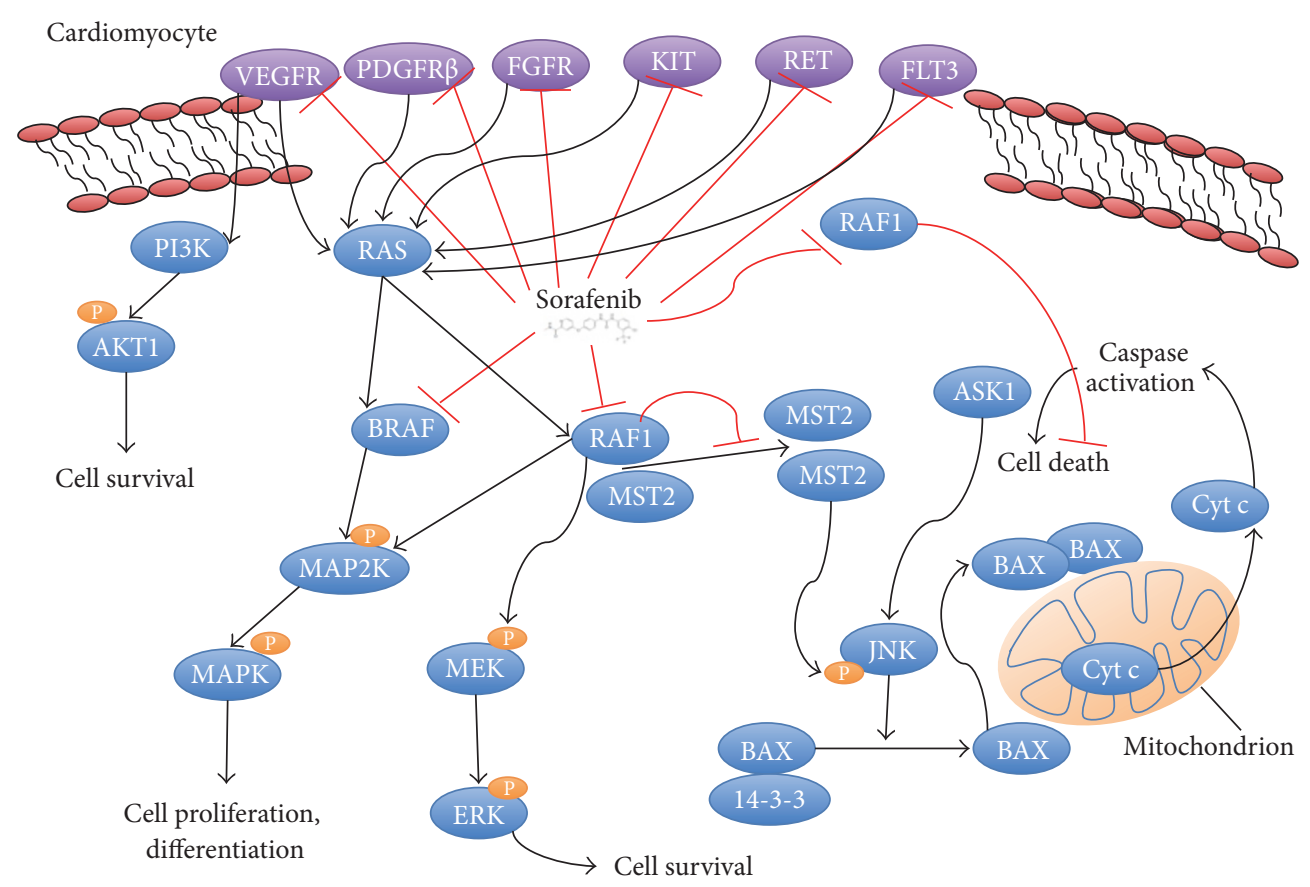

FIGURE 5: Sorafenib inhibits multiple tyrosine kinase and second messenger pathways. In the cardiac myocyte, inhibition of prosurvival pathways RAS/MAPK via BRAF and P13K/AKT1 via VEGFR leads to diminished survival. Sorafenib further inhibits RAF1, which leads to loss of tonic inhibition of cell death pathways and death through activation of caspases.

treatment of renal cell carcinoma (RCC) with sorafenib, $68 \%$ had some form of cardiovascular toxicity, with most cases (59\%) having worsened hypertension. Newly diagnosed heart failure, defined as an elevation in NT-proBNP and a decline in LVEF, occurred in $40 \%$ of patients on sunitinib and in $4 \%$ of patients on sorafenib [2]. In a meta-analysis of 21 randomized controlled trials with patients undergoing treatment of HCC, there was a 2.7-fold greater heart failure risk in those that received TKIs compared to those who did not (2.39\% versus $0.75 \%, p<0.001$ ) [23].

Therapeutic Challenges and Considerations in the Treatment of Hypertension. Hepatocellular carcinoma is one of the most common cancers in the world and typically presents in advanced stages. Standard treatments during early stages include partial hepatectomy, local ablation, and liver transplantation. Sorafenib is recommended as a first-line drug for the treatment of advanced HCC $[1,2]$.

In the case of sorafenib-induced hypertension, blood pressure is treated with conventional therapy and appears not to impact or worsen progression-free survival among patients treated for metastatic RCC [24]. Early intervention of hypertension is essential to reduce subsequent risks of developing cardiomyopathy and heart failure. The Angiogenesis Task Force of the National Cancer Institute Investigational Drug Steering Committee recommends regular blood pressure monitoring before treatment as well as during treatment, weekly during the first cycle and then every 2-3 weeks, with a goal BP of less than $140 / 90 \mathrm{mmHg}$ for most patients and a goal BP of less than 130/80 for those with diabetes or chronic kidney disease [25]. Sorafenib is also associated with the development of proteinuria, and it is recommended that urine protein be tested before and after treatment initiation. When patients develop proteinuria, clinicians should strive to control blood pressure with either angiotensin-converting enzyme inhibitors (ACE-I) or dihydropyridine calcium channel blockers (CCB), which have been recommended as firstand second-line agents (Figure 6) [26]. Treatment with ACEI is contraindicated if patients develop hyperkalemia, severe cough, or angioedema or have advanced chronic kidney disease. Transitioning to angiotensin receptor blocker is possible in those patients intolerant to ACE-I though clinical data is limited. Discontinuation of sorafenib can be considered in patients with resistant hypertension.

Therapeutic Challenges and Considerations in the Treatment of Systolic Heart Failure. Although it is typically recommended that chemotherapy be discontinued when significant heart failure develops, case reports have shown that sorafenib can be used to treat advanced HCC for as long as 12 months and may be reduced in those that develop heart failure [1]. It is difficult to know which heart failure medications would benefit patients exposed to TKIs. Very few clinical studies have investigated the treatment of heart failure due to TKIs in a prospective fashion. Newer generation TKIs are less selective than their predecessors, and while the cancer cell growth is strongly halted, the potential for cardiac toxicity is higher. At this time there are no medications that specifically target and reverse TKI-related cardiotoxicity. The ACC/AHA guidelines recommend that patients receiving 


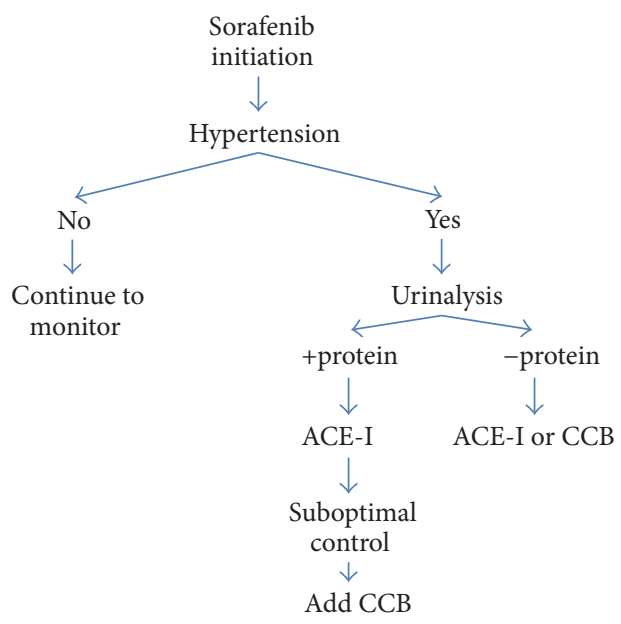

FIGURE 6: Algorithm for treatment of sorafenib-induced hypertension.

chemotherapeutic agents should be monitored carefully. A baseline echocardiogram prior to treatment with TKIs in patients with cardiac risk factors and close follow-up in monitoring for heart failure symptoms is appropriate $[27,28]$.

In patients that develop clinical heart failure or systolic dysfunction on echocardiography, treatment with a combination of guideline directed ACE-I, and beta-blockers have been shown to reduce heart failure-associated mortality and improve cardiovascular morbidity [27]. The most well studied agents in the treatment of anthracycline-induced cardiomyopathy are carvedilol and enalapril though the mechanism, severity, and toxicity of anthracyclines differ from the cardiotoxicity of TKIs [29, 30]. Aldosterone antagonists have also been shown to further reduce mortality in advanced heart failure in patients with systolic heart failure, LVEF < $35 \%$, and NYHA III-IV. Treatment of salt and water retention in systolic heart failure is further treated with loop diuretics. Implantable defibrillators used as primary prevention for sudden cardiac death are recommended in those with greater than a 1-year life expectancy with persistently severe LV dysfunction for greater than three months despite optimal medical therapy. In patients that develop heart failure symptoms and successfully complete treatment with TKIs, followup echocardiography is appropriate to assess for reversal of LV dysfunction [26].

Dobutamine Treatment May Be Considered in Those with Severe Heart Failure. Baseline diminished contractile reserve with subsequent exposure to sorafenib led to the development of cardiogenic shock in our patient who was subsequently treated with dobutamine, an inotrope with weaker chronotropic effects, which successfully restored a normal hemodynamic profile. While it is well known that inotropic support is associated with adverse long-term cardiovascular outcomes, the clinical efficacy of inotropes after exposure to cardiac toxic medications is unknown. Dobutamine's pharmacologic function does not depend on tyrosine kinases but rather on G-protein coupled receptors. Whether sensitivity to beta-1 adrenergic activation by dobutamine is increased after tyrosine kinase inhibition is unknown. In response to isoproterenol, the presence of genistein was shown to potentiate beta-adrenergic sensitivity in the heart of animal models [31].

\section{Conclusion}

In our patient with underlying ischemic cardiomyopathy and advanced hepatocellular carcinoma, the management of heart failure in the setting of coexisting malignancy poses interesting and new clinical challenges. Sorafenib is a tyrosine kinase inhibitor that targets multiple tyrosine kinase receptors involved in angiogenesis and tumor growth. Unfortunately, sorafenib-associated cardiotoxic effects such as hypertension, reduced LVEF, and heart failure are of great concern and contribute to the challenging nature of HCC management. It is essential that TKI-induced hypertension be treated aggressively with standard treatments and regular monitoring. Guidelines also recommend echocardiogram monitoring for patients started on chemotherapeutic agents, especially those with cardiac risk factors, prior to treatment initiation, once heart failure symptoms develop, and after treatment completion. There are currently no specific recommendations for managing TKI-induced heart failure, and patients are typically managed with standard heart failure treatments. Beta-adrenergic effects of dobutamine may actually be potentiated by tyrosine kinase inhibition.

We initially considered more common causes for heart failure exacerbation in our patient. Patients with restrictive heart failure often develop worsened heart failure symptoms due to sustained periods of atrial fibrillation with rapid ventricular response. Prior to hospitalization, however, the patient's ventricular rate was well controlled with beta blockade, and it is our belief that the interval decline in ventricular function led to increased left atrial pressure and presentation of atrial fibrillation with rapid ventricular response. Furthermore, there were no EKG abnormalities 
suggestive of myocardial infarction or evidence of severe valvular regurgitation on echocardiography. There was the possibility that overdiuresis due to an inability to accurately estimate the filling pressures on physical exam led to inadequate preload leading to low cardiac output shock. However, the patient's right heart catheterization reflected that the filling pressures remained elevated after diuresis and that the etiology of low cardiac output was mainly attributed to diminished contractility.

After successful titration of guideline directed treatment and discontinuation of dobutamine, a repeat echo demonstrated baseline LV function. A repeat trial of sorafenib at a lower dose was offered to the patient but was declined. Although sorafenib is the potential predisposing factor for the patient's decompensation, the causal relationship between this drug and heart failure is still unclear since rechallenge was not performed. The patient was discharged home with palliative care follow-up for coordination of hospice.

\section{Abbreviations}

ACC: $\quad$ American College of Cardiology

ACE-I: Angiotensin-converting enzyme inhibitors

AFP: $\quad$ Alpha-fetoprotein

AHA: $\quad$ American Heart Association

ASK1: $\quad$ Apoptosis signal-regulating kinase 1

BP: $\quad$ Blood pressure

BPM: $\quad$ Beats per minute

CCB: $\quad$ Calcium channel blocker

EGFR: $\quad$ Epidermal growth factor receptor

ERK: $\quad$ Extracellular signal regulated kinase

HCC: Hepatocellular carcinoma

HER2: Human epidermal growth factor receptor

JVP: Jugular venous pressure

LV: $\quad$ Left ventricle

LVEF: $\quad$ Left ventricular ejection fraction

MAP: $\quad$ Mean arterial pressure

MAPK: $\quad$ Mitogen-activated protein kinase

MST2: $\quad$ Mammalian sterile 20 kinase 2

MRI: $\quad$ Magnetic resonance imaging

NT-proBNP: N-terminal prohormone of brain natriuretic peptide

NYHA: New York Heart Association

PA: $\quad$ Pulmonary artery

PCWP: Pulmonary capillary wedge pressure

RAF1: Rapidly accelerated fibrosarcoma

RCC: $\quad$ Renal cell carcinoma

RHC: $\quad$ Right heart catheterization

RV: $\quad$ Right ventricle

TACE: Transcatheter arterial chemoembolization

TKI: $\quad$ Tyrosine kinase inhibitor

TKR: $\quad$ Tyrosine kinase receptor

VEGF: Vascular endothelial growth factor

VEGFR: Vascular endothelial growth factor receptor

WHO: World Health Organization.

\section{Conflicts of Interest}

The authors declare that they have no conflicts of interest.

\section{References}

[1] C. Valsuani, O. Siclari, A. Camerini et al., "Sorafenib in a patient with advanced hepatocellular carcinoma and serious impairment of left ventricular function: a case report," Cases Journal, vol. 2, no. 12, article no. 9133, 2009.

[2] P. S. Hall, L. C. Harshman, S. Srinivas, and R. M. Witteles, "The frequency and severity of cardiovascular toxicity from targeted therapy in advanced renal cell carcinoma patients," JACC: Heart Failure, vol. 1, no. 1, pp. 72-78, 2013.

[3] J. M. Llovet, S. Ricci, V. Mazzaferro et al., "Sorafenib in advanced hepatocellular carcinoma," The New England Journal of Medicine, vol. 359, no. 4, pp. 378-390, 2008.

[4] G. Condorelli, A. Drusco, G. Stassi et al., "Akt induces enhanced myocardial contractility and cell size in vivo in transgenic mice," Proceedings of the National Academy of Sciences of the United States of America, vol. 99, no. 19, pp. 12333-12338, 2002.

[5] T. Yamazaki, I. Komuro, and Y. Yazaki, "Signalling pathways for cardiac hypertrophy," Cellular Signalling, vol. 10, no. 10, pp. 693698, 1998.

[6] M. A. Cobleigh, C. L. Vogel, D. Tripathy et al., "Multinational study of the efficacy and safety of humanized anti-HER2 monoclonal antibody in women who have HER2-overexpressing metastatic breast cancer that has progressed after chemotherapy for metastatic disease," Journal of Clinical Oncology, vol. 17, no. 9, pp. 2639-2648, 1999.

[7] M. Rossi, G. Carioli, M. Bonifazi et al., "Trastuzumab for HER2+ metastatic breast cancer in clinical practice: cardiotoxicity and overall survival," European Journal of Cancer, vol. 52, pp. 41-49, 2016.

[8] M. Bonifazi, M. Franchi, M. Rossi et al., "Trastuzumab-related cardiotoxicity in early breast cancer: a cohort study," Oncologist, vol. 18, no. 7, pp. 795-801, 2013.

[9] M. Procter, T. M. Suter, E. de Azambuja et al., "Longer-term assessment of trastuzumab-related cardiac adverse events in the Herceptin Adjuvant (HERA) trial," Journal of Clinical Oncology, vol. 28, no. 21, pp. 3422-3428, 2010.

[10] X. Pivot, T. Suter, J. M. Nabholtz et al., "Cardiac toxicity events in the PHARE trial, an adjuvant trastuzumab randomised phase III study," European Journal of Cancer, vol. 51, no. 13, article 9505, pp. 1660-1666, 2015.

[11] E. De Azambuja, M. J. Procter, D. J. Van Veldhuisen et al., "Trastuzumab-associated cardiac events at 8 years of median follow-up in the herceptin adjuvant trial (BIG 1-01)," Journal of Clinical Oncology, vol. 32, no. 20, pp. 2159-2165, 2014.

[12] T. Force, D. S. Krause, and R. A. Van Etten, "Molecular mechanisms of cardiotoxicity of tyrosine kinase inhibition," Nature Reviews Cancer, vol. 7, no. 5, pp. 332-344, 2007.

[13] O. F. Bueno, L. J. De Windt, K. M. Tymitz et al., “The MEK1ERK1/2 signaling pathway promotes compensated cardiac hypertrophy in transgenic mice," The European Molecular Biology Organization Journal, vol. 19, pp. 6341-6350, 2000.

[14] B. B. Hasinoff, D. Patel, and X. Wu, "The dual-targeted HER1/ HER2 tyrosine kinase inhibitor lapatinib strongly potentiates the cardiac myocyte-damaging effects of doxorubicin," Cardiovascular Toxicology, vol. 13, no. 1, pp. 33-47, 2013.

[15] O. Yamaguchi, T. Watanabe, K. Nishida et al., "Cardiac-specific disruption of the c-raf-1 gene induces cardiac dysfunction and apoptosis," The Journal of Clinical Investigation, vol. 114, no. 7, pp. 937-943, 2004.

[16] I. S. Harris, S. Zhang, I. Treskov, A. Kovacs, C. Weinheimer, and A. J. Muslin, "Raf-1 kinase is required for cardiac hypertrophy 
and cardiomyocyte survival in response to pressure overload," Circulation, vol. 110, no. 6, pp. 718-723, 2004.

[17] H. Park, S.-H. Han, H. Kang, and K. Park, "A case of reversible cardiomyopathy associated with sorafenib in advanced hepatocellular carcinoma," International Journal of Cardiology, vol. 160, no. 1, pp. e10-e11, 2012.

[18] S. Rohrbach, X. Yan, E. O. Weinberg et al., "Neuregulin in cardiac hypertrophy in rats with aortic stenosis: differential expression of erbB2 and erbB4 receptors," Circulation, vol. 100, no. 4, pp. 407-412, 1999.

[19] A. M. J. Thijs, C. M. L. Van Herpen, F. C. G. J. Sweep et al., "Role of endogenous vascular endothelial growth factor in endothelium-dependent vasodilation in humans," Hypertension, vol. 61, no. 5, pp. 1060-1065, 2013.

[20] J.-J. Mourad, G. des Guetz, H. Debbabi, and B. I. Levy, "Blood pressure rise following angiogenesis inhibition by bevacizumab. A crucial role for microcirculation," Annals of Oncology, vol. 19, no. 5, pp. 927-934, 2008.

[21] W.-X. Qi, S. Fu, Q. Zhang, and X.-M. Guo, "Bevacizumab increases the risk of severe congestive heart failure in cancer patients: an up-to-date meta-analysis with a focus on different subgroups," Clinical Drug Investigation, vol. 34, no. 10, pp. 681690, 2014.

[22] C. P. Escalante, Y. C. Chang, K. Liao et al., "Meta-analysis of cardiovascular toxicity risks in cancer patients on selected targeted agents," Supportive Care in Cancer, vol. 24, no. 9, pp. 4057-4074, 2016.

[23] P. Ghatalia, C. J. Morgan, Y. Je et al., "Congestive heart failure with vascular endothelial growth factor receptor tyrosine kinase inhibitors," Critical Reviews in Oncology/Hematology, vol. 94, no. 2, pp. 228-237, 2015.

[24] S. Szmit, P. Langiewicz, J. Zlnierek, P. Nurzynski, M. Zaborowska, K. J. Filipiak et al., "Hypertension as a predictive factor for survival outcomes in patients with metastatic renal cell carcinoma treated with sunitinib after progression on cytokines.," in Kidney And Blood Pressure Research, vol. 35, pp. 18-25, 2012.

[25] D. J. Lenihan and P. R. Kowey, "Overview and management of cardiac adverse events associated with tyrosine kinase inhibitors," Oncologist, vol. 18, no. 8, pp. 900-908, 2013.

[26] B. Ky, P. Vejpongsa, E. T. H. Yeh, T. Force, and J. J. Moslehi, "Emerging paradigms in cardiomyopathies associated with cancer therapies," Circulation Research, vol. 113, no. 6, pp. 754764, 2013.

[27] W. C. Yancy, M. Jessup, B. Bozkurt et al., "Guideline for the management of heart failure," Circulation, vol. 128, pp. e240e327, 2013.

[28] R. C. Hendel, M. R. Patel, C. M. Kramer, M. Poon, J. C. Carr, N. A. Gerstad et al., "ACCF/ACR/SCCT/SCMR/ASNC/NASCI/ SCAI/SIR 2006 appropriateness criteria for cardiac computed tomography and cardiac magnetic resonance imaging: a report of the American College of Cardiology Foundation Quality Strategic Directions Committee Appropriateness Criteria Working Group, American College of Radiology, Society of Cardiovascular Computed Tomography, Society for Cardiovascular Magnetic Resonance, American Society of Nuclear Cardiology, North American Society for Cardiac Imaging, Society for Cardiovascular Angiography and Interventions, and Society of Interventional Radiology," Journal of the American College of Cardiology, vol. 48, pp. 1475-1497, 2006.
[29] Y.-L. Chen, S.-Y. Chung, H.-T. Chai et al., "Early administration of carvedilol protected against doxorubicin-induced cardiomyopathy," Journal of Pharmacology and Experimental Therapeutics, vol. 355, no. 3, pp. 516-527, 2015.

[30] G. Janbabai, M. Nabati, M. Faghihinia, S. Azizi, S. Borhani, and J. Yazdani, "Effect of enalapril on preventing anthracyclineinduced cardiomyopathy," Cardiovascular Toxicology, pp. 1-10, 2016.

[31] L. C. Hool, L. M. Middleton, and R. D. Harvey, "Genistein increases the sensitivity of cardiac ion channels to $\beta$-adrenergic receptor stimulation," Circulation Research, vol. 83, no. 1, pp. 3342, 1998. 


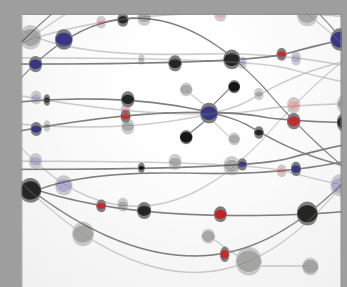

The Scientific World Journal
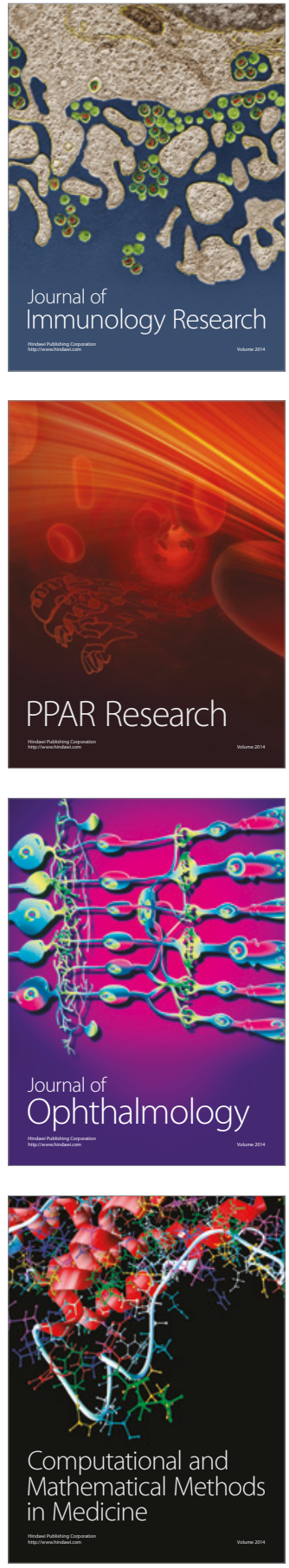

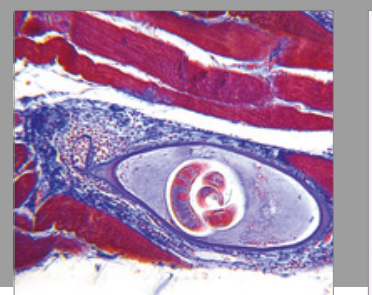

Gastroenterology Research and Practice
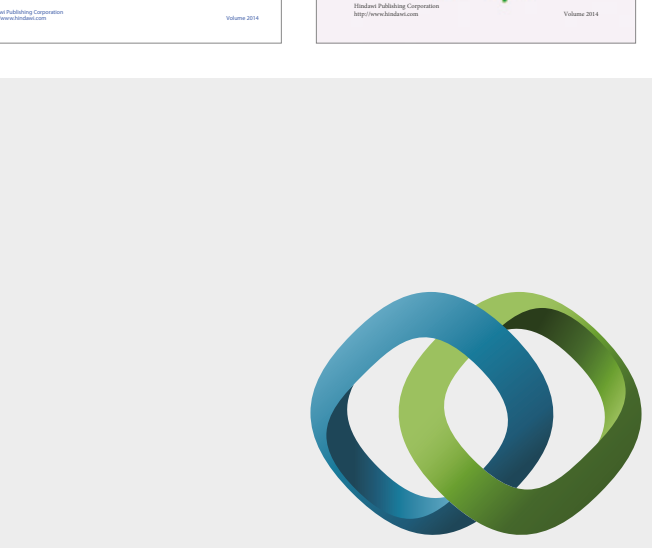

\section{Hindawi}

Submit your manuscripts at

https://www.hindawi.com
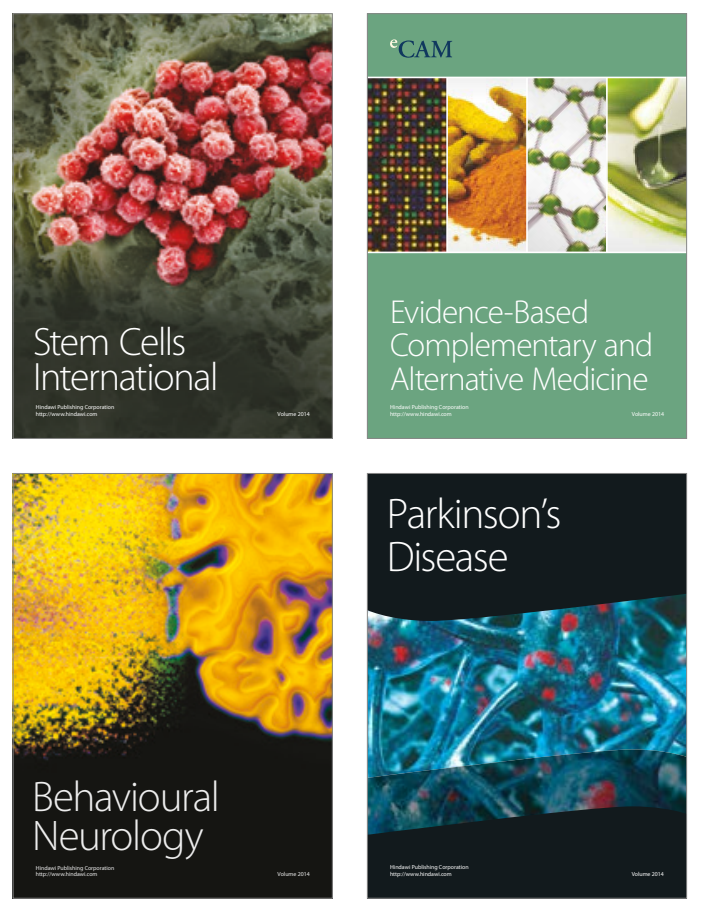
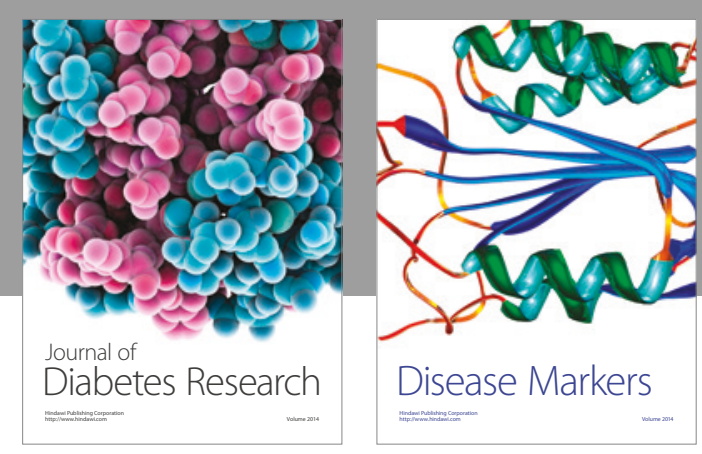

Disease Markers
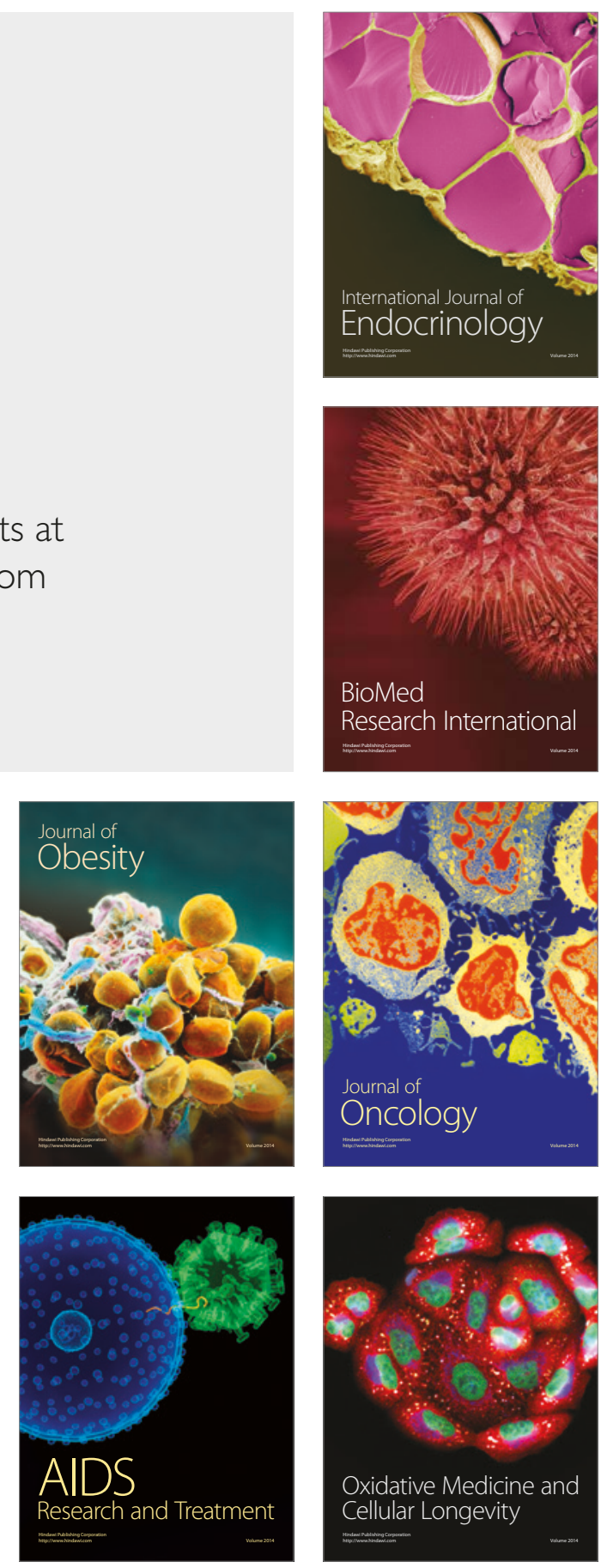